\title{
ANÁLISE DA CINÉTICA DE SECAGEM E ISOTERMAS DE SORÇÃO DE FILMES DE PECTINA INCORPORADOS COM MESOCARPO DE COCO BABAÇU
}

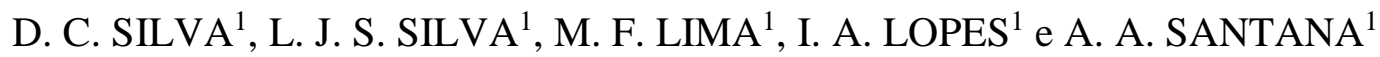 \\ ${ }^{1}$ Universidade Federal do Maranhão, Centro de Ciências Exatas e suas Tecnologias, \\ Departamento de Engenharia Química \\ E-mail para contato: dennys.correia@hotmail.com, audirene.amorim@gmail.com
}

\begin{abstract}
RESUMO - O consumidor consciente tem dado preferência a alimentos processados de melhor qualidade nutricional e com vida de prateleira prolongada, sempre com a devida preocupação com os efeitos prejudiciais acumulativos ao meio ambiente do descarte de subprodutos. Filmes biodegradáveis estão inseridos neste contexto. Eles são produzidos a partir de polímeros naturais, principalmente polissacarídeos e proteínas, com potencial aplicação na indústria farmacêutica e alimentícia. A incorporação do mesocarpo de coco babaçu em filmes de pectina além de conferir maior resistência ao filme, proporciona melhores propriedades físico-químicas. Portanto, o objetivo deste trabalho foi produzir e caracterizar os filmes de pectina incorporados de mesocarpo de coco babaçu (MCB), onde os filmes foram obtidos pela técnica casting e após análise estatística por Delineamento de Composto Central Rotacional, foram escolhidas as melhores formulações para produção de sacolas biodegradáveis e colocadas sobre teste de cinética de secagem e isoterma de sorção. Os resultados confirmaram que os biofilmes se apresentaram bastante solúveis, com boa biodegradação.
\end{abstract}

\section{INTRODUÇÃO}

Filmes e recobrimentos biodegradáveis são aqueles formados a partir de polímeros naturais, de origem animal ou vegetal, como polissacarídeos, lipídios e proteínas e que quando lançados no meio ambiente, convertem-se em compostos simples, mineralizados, que, redistribuídos através dos ciclos de carbono, nitrogênio e enxofre não agridem o biossistema. Existem alguns requisitos específicos para o seu uso, tais como: boa aceitabilidade sensorial, propriedades de barreira e mecânicas adequadas, estabilidade bioquímica, físico-química e microbiológica, ser inócuo, não poluente, de processamento simples e de baixo custo (Santana e Kieckbusch, 2013).

A aplicação mais conhecida da pectina é como gelificante ou estabilizante, mas também características como biocompatibilidade e a não-toxicidade também permitem que a pectina esteja sendo crescentemente utilizada nas áreas farmacêutica e biotecnológica (Liu et al., 2007). Já o babaçu (Orbignya phalerata Mart.) é uma das mais importantes representantes das palmeiras brasileiras, onde nos estados do Maranhão, Piauí e Tocantins que se concentram as maiores extensões de matas onde predominam os babaçus (Carraza et al., 2012). O mesocarpo de coco babaçu (MCB) pode ser utilizado na alimentação humana por possui várias fontes nutricionais. Na literatura não há relatos sobre o uso do mesocarpo de coco babaçu como 
matriz na produção de biopolímeros. Outro componente importante na elaboração de filmes biodegradáveis é o plastificante, uma pequena molécula de baixa volatilidade que, quando adicionado aos materiais poliméricos, modifica a sua organização tridimensional, diminui as forças intermoleculares e aumento de volume livre além da mobilidade da cadeia. Essas mudanças na organização molecular são causadas pelos resultados dos plastificante em aumentar a extensibilidade e a flexibilidade da película, enquanto a coesão e a rigidez dos filmes são reduzidas. Portanto, o objetivo desse trabalho foi, além de definir as formulações adequadas de produção de biofilmes para confecção de sacolas, realizar análises de cinética de secagem e isotermas de sorção para verificar as características dos mesmos.

\section{MATERIAS E MÉTODOS}

\subsection{Matérias-Primas}

Para a confecção dos filmes, foi utilizado pectina de baixa metoxilação (Cpkelco, Limeira, Brasil), coco babaçu disponibilizado pela flora da Universidade Federal do Maranhão (UFMA), cloreto de cálcio dihidratado (Merck - Alemanha) (agente reticulante) e glicerol (Synth - Brasil).

\subsection{Elaboração Dos Filmes}

Os filmes foram confeccionados de acordo com metodologia estabelecida por Santana (2010), sendo obtidos segundo a técnica de casting, a qual consiste na preparação da solução filmogênica e aplicação conveniente da mesma em um suporte (placas de estireno, $\mathrm{d}=15 \mathrm{~cm}$ ). A secagem dos filmes foi a $40^{\circ} \mathrm{C}$ em estufa com recirculação de ar (Nova Ética, 400 1ND, Brasil) durante 22-24 horas. Em seguida, foram analisados os filmes ideais para confecção de sacolas pelo Delineamento Composto Central Rotacional (DCCR) (Tabela 1). As variáveis independentes foram: concentração de pectina, MCB e glicerol e as respostas analisadas pelo software Statistica 9.0 (StatSoft, Inc., USA). Filmes de alta umidade e baixa espessura foram considerados próprios para o fim.

Tabela 1 - Formulação dos ensaios para confecção dos biofilmes

\begin{tabular}{|c|c|c|c|c|c|}
\hline Variáveis & $-1,68$ & -1 & 0 & +1 & $-1,68$ \\
\hline \hline Pectina $(\mathrm{g})$ & 2 & 4,4 & 6 & 8,4 & 10 \\
\hline MCB $(\mathrm{g})$ & 0 & 1,5 & 2,5 & 4 & 5 \\
\hline Glicerol $(\mathrm{ml})$ & 1 & 2,2 & 3 & 4,2 & 5 \\
\hline
\end{tabular}

\subsection{Cinética de Secagem}

Pedaços de filme contendo a solução polimérica foram pesados em intervalos de tempo predefinidos durante o processo de secagem (controle gravimétrico), a fim de avaliar a secagem cinética para cada condição. Os pedaços foram secos até atingir peso constante (estado de equilíbrio), observando-se diferentes tempos de secagem. O teor de umidade final dos filmes foi determinado no final do processo de secagem. Utilizou-se como ferramenta a regressão não linear dos dados experimentais e os modelos de Weibull (Equação 1), Peleg (Equação 2), Lewis (Equação 3), Logarítmico (Equação 4) foram estudados, o qual possui dependência da Equação de umidade relativa RU (Equação 5). 


$$
\begin{aligned}
& R U=\exp \left(-\frac{t}{\beta}\right) \\
& X=X_{o}-\frac{t}{k_{1}+k_{2} t} \\
& R U=\exp (-k t) \\
& R U=a \cdot \exp (-k t)+c \\
& R U=\frac{X-X_{\theta}}{X_{o}-X_{\theta}}
\end{aligned}
$$

Em que $X$ é o teor de umidade (base seca) (g água/g); $X_{\varepsilon}$ é o teor de umidade de equilíbrio (base seca) (g água/g); $X_{o}$ é o teor de umidade inicial (base seca) (g água/g).

\subsection{Isotermas de Sorção}

A umidade de equilíbrio dos filmes, após determinação dos ideais para sacolas, sob diferentes condições de atividades de água foi avaliada pelo método gravimétrico estático (Rizvi, 1986). Foram utilizados 6 sais $\left(\mathrm{MgCl}_{2}, \mathrm{LiCl}, \mathrm{K}_{2} \mathrm{CO}_{3}, \mathrm{KI}, \mathrm{NaCl}, \mathrm{CH}_{3} \mathrm{COOK}\right)$ preparados em soluções saturadas com intervalo de atividade de água correspondente variando entre 0,112 e 0,689 .

Todas as soluções foram preparadas e transferidas para recipientes hermeticamente fechados e as amostras, com aproximadamente $1 \mathrm{~g}$ de filme foram colocadas dentro de cadinhos de alumínio e acondicionados nos potes herméticos que foram postos em temperatura ambiente $\left(25^{\circ} \mathrm{C}\right)$. A pesagem das amostras ocorreu semanalmente até o equilíbrio. Após o equilíbrio, os cadinhos foram colocados em uma estufa à $105^{\circ} \mathrm{C}$, com o objetivo de determinar a massa seca para o cálculo da umidade de equilíbrio. Para os ajustes das isotermas de dessorção, utilizou-se como ferramenta a regressão não linear dos dados experimentais e os modelos de GAB (Equação 6), Oswin (Equação 7), BET (Equação 8) e Halsey (Equação 9 foram estudados. Os valores dos desvios relativos médio, juntamente com os parâmetros das equações foram obtidos pelo programa computacional statistica (versão 9.0) utilizando-se o módulo de estimativa não linear (método de conversão Simplex-Quase Newton.

$$
\begin{aligned}
& X_{e}=\frac{X_{m} C K a_{w}}{\left(1-K a_{w}\right)\left(1-K a_{w}+C K a_{w}\right)} \\
& X_{e}=a\left(\frac{a_{w}}{1-a_{w}}\right)^{b} \\
& X_{\varepsilon}=\frac{a_{w}}{\left(1-a_{w}\right)\left(b a_{w}+c\right)}
\end{aligned}
$$




$$
a_{w}=\exp \left(\frac{-A}{X_{e}^{r}}\right)
$$

\section{RESULTADOS E DISCUSSÃO}

Através das análises estatísticas, tem-se duas novas formulações ideais para a confecção de sacolas através dos primeiros ensaios, uma com alta quantidade de glicerol (F1) e outra com baixa (F2). Nessas formulações, aumentou-se as concentrações de pectina e MCB e variou-se as concentrações de glicerol de modo que a umidade teve valores altos e a espessura dos filmes valores altos.

\subsection{Cinética de Secagem}

A Figura 1 mostra as curvas de secagem dos filmes F1 e F2, respectivamente, em diferentes temperaturas de acordo com a equação de Peleg, que foi a que melhor se ajustou nas duas Formulações.

Figura 1 - Valores experimentais e estimados de razão de umidade pela estimativa dos parâmetros da equação de Peleg para a F1(a) e F2(b). Legenda: $(\bullet) 50^{\circ} \mathrm{C},(\boldsymbol{\Delta}) 60^{\circ} \mathrm{C},(\boldsymbol{\square})$ $70^{\circ} \mathrm{C}$ e (一) Modelo de Peleg.

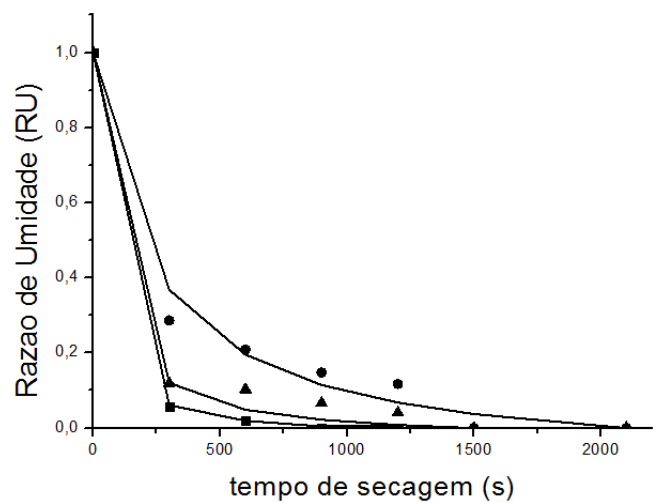

(a)

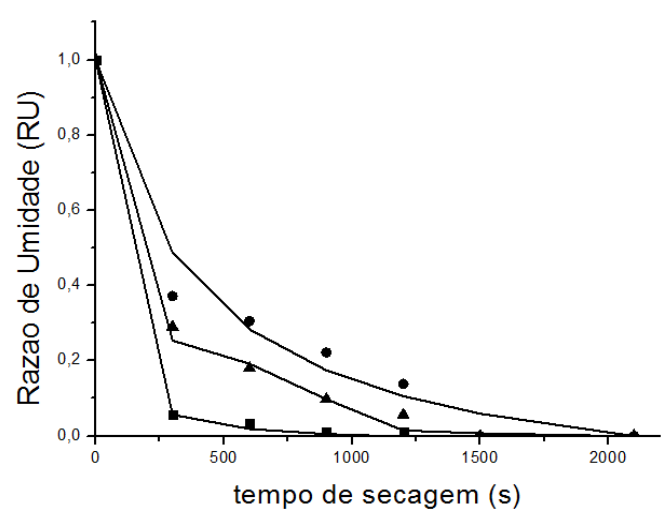

(b)

Observa-se a influência da temperatura do ar na cinética do processo e como previsto, o tempo de secagem decresce com o aumento da temperatura do ar. $\mathrm{Na}$ faixa de $70^{\circ} \mathrm{C}$, o comportamento da curva nas duas formulações possui o mesmo comportamento, mas nas faixas de 50 e $60^{\circ} \mathrm{C}$ nota-se uma razão de umidade maior em F2 do que em F1 nos mesmos intervalos de secagem. Isso pode ser explicado pela ausência de glicerol em F2 em proporções menores que em F1, sendo essas faixas ocupadas por um teor maior de umidade. O modelo de Peleg possui valores de MRE menores em relação ao modelo de Weibull, mas em relação ao coeficiente de determinação $\left(\mathrm{R}^{2}\right)$, a equação de Weibull apresentou valores tão elevados quanto a equação de Peleg, estando, para todos os tratamentos, acima de $98 \%(0,98)$ o que, segundo Madamba et al. (1996) significa um bom ajuste dos modelos para representação do fenômeno de secagem.

Apresentam-se, nas Tabela 2, os resumos do ajuste dos modelos por meio de regressão não linear aos dados experimentais de secagem dos filmes, considerando-se as diferentes temperaturas do ar, com os parâmetros ajustados, o erro relativo médio de ajuste (MRE) e o coeficiente de determinação $\left(\mathrm{R}^{2}\right)$ de cada modelo. 
Tabela 2 - Parâmetros obtidos, valores do coeficiente de determinação $\left(\mathrm{R}^{2}\right)$ e do erro médio estimado (MRE), calculados para verificação do ajuste dos modelos matemáticos aos valores experimentais da secagem dos filmes F1 e F2 obtidos nas temperaturas de 50, 60 e $70{ }^{\circ} \mathrm{C}$.

\begin{tabular}{|c|c|c|c|c|c|c|c|}
\hline \multirow{2}{*}{$\begin{array}{c}\text { Modelos } \\
\text { Matemáticos } \\
\end{array}$} & \multirow[t]{2}{*}{ Parâmetros } & \multicolumn{3}{|c|}{$\mathbf{F} 1\left(\mathbf{T}^{\circ} \mathbf{C}\right)$} & \multicolumn{3}{|c|}{$\mathbf{F} 2\left(\mathbf{T}^{\circ} \mathrm{C}\right)$} \\
\hline & & 50 & 60 & 70 & 50 & 60 & 70 \\
\hline \multirow{3}{*}{ Weibull } & $\beta$ & 183,326 & 141,326 & 105,166 & 148,794 & 195,444 & 104,468 \\
\hline & $\operatorname{MRE}(\%)$ & 9,396 & 12,409 & 3,654 & 6,614 & 7,374 & 3,091 \\
\hline & $R^{2}$ & 0,953 & 0,991 & 0,999 & 0,947 & 0,978 & 0,999 \\
\hline \multirow[t]{4}{*}{ Peleg } & $k_{1}$ & 90,520 & 32,170 & 15,916 & 48,480 & 31,463 & 12,401 \\
\hline & $\boldsymbol{k}_{2}$ & 0,653 & 0,606 & 0,570 & 0,629 & 0,700 & 0,497 \\
\hline & $\operatorname{MRE}(\%)$ & 9,738 & 8,487 & 3,102 & 5,240 & 5,240 & 2,477 \\
\hline & $R^{2}$ & 0,983 & 0,996 & 0,999 & 0,973 & 0,934 & 0,999 \\
\hline \multirow[t]{3}{*}{ Lewis } & $\boldsymbol{k}$ & $\begin{array}{c}7,076 \mathrm{E}- \\
03\end{array}$ & $\begin{array}{c}2,610 \mathrm{E}- \\
03\end{array}$ & $\begin{array}{c}9,509 \mathrm{E}- \\
03\end{array}$ & $\begin{array}{c}1,822 \mathrm{E}- \\
03\end{array}$ & $\begin{array}{c}5,117 \mathrm{E}- \\
03\end{array}$ & $\begin{array}{c}9,572 \mathrm{E}- \\
03\end{array}$ \\
\hline & $\operatorname{MRE}(\%)$ & 9,396 & 12,409 & 3,654 & 6,635 & 7,374 & 5,298 \\
\hline & $R^{2}$ & 0,953 & 0,991 & 0,999 & 0,946 & 0,978 & 0,997 \\
\hline \multirow[t]{4}{*}{ Page } & $\boldsymbol{k}$ & $\begin{array}{c}6,628 \mathrm{E}- \\
03 \\
\end{array}$ & $\begin{array}{c}7,365 \mathrm{E}- \\
03 \\
\end{array}$ & $\begin{array}{c}5,533 \mathrm{E}- \\
03 \\
\end{array}$ & $\begin{array}{c}9,103 \mathrm{E}- \\
02 \\
\end{array}$ & $\begin{array}{c}7,535 \mathrm{E}- \\
02 \\
\end{array}$ & $\begin{array}{c}5,103 \mathrm{E}- \\
02 \\
\end{array}$ \\
\hline & $n$ & 0,85 & 0,99 & 1,09 & 1,25 & 1,25 & 0,71 \\
\hline & $\operatorname{MRE}(\%)$ & 8,443 & 12,386 & 3,860 & 6,615 & 7,374 & 4,327 \\
\hline & $R^{2}$ & 0,969 & 0,991 & 0,999 & 0,947 & 0,978 & 0,999 \\
\hline \multirow[t]{5}{*}{ Logarítmico } & $a$ & 0,676 & 0,732 & 0,935 & 0,554 & 0,663 & 0,948 \\
\hline & $\boldsymbol{k}$ & 9,55 & $\begin{array}{c}3,49 \mathrm{E}- \\
03\end{array}$ & $\begin{array}{c}9,50 \mathrm{E}- \\
03\end{array}$ & 1,28 & 1,00 & 1,00 \\
\hline & $\boldsymbol{c}$ & 0,32 & 0,13 & 0,06 & 0,45 & 0,34 & 0,05 \\
\hline & $\operatorname{MRE}(\%)$ & 8,577 & 15,877 & 3,679 & 3,260 & 9,297 & 3,509 \\
\hline & $R^{2}$ & 0,910 & 0,939 & 0,999 & 0,829 & 0,879 & 0,999 \\
\hline
\end{tabular}

\subsection{Isotermas de Sorção}

A Figura 2 mostras as isotermas adquiridas nas duas formulações enquanto a Tabela 3 mostra todos os parâmetros obtidos para os modelos experimentais, os coeficientes de determinação $\left(R^{2}\right)$ e os erros médios relativos de cada ajuste para as Formulações 1 e 2.

Figura 2 - Relação umidade de equilíbrio e atividade de água ( $\left.a_{w}\right)$ para a F1 e F2. Legenda:

$(\bullet)$ dados experimentais e (一) Modelo de Oswin.

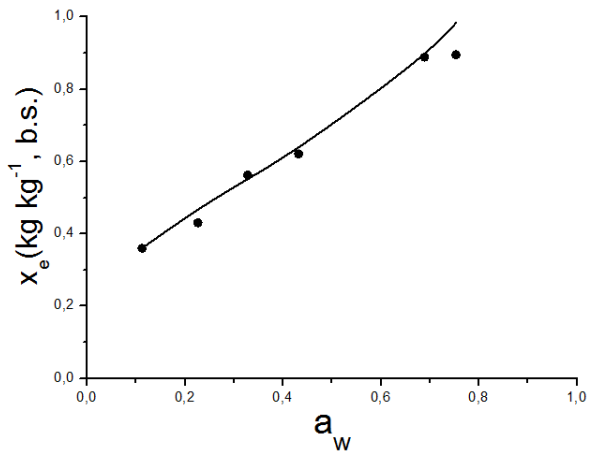

(a)

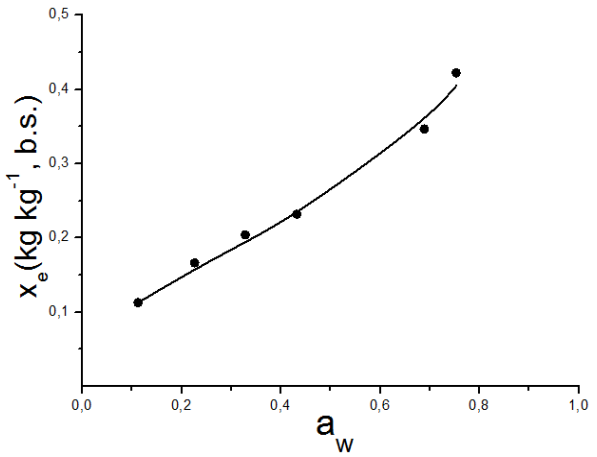

(b) 
Os filmes adsorvem relativamente alta quantidade de água a altas atividades de água e grande quantidade de água a altas umidades relativas. $\mathrm{O}$ modelo que melhor se adequou ao experimento foi o de Oswin, mostrando como a umidade de equilíbrio decresce variação da temperatura. Isto acontece devido à energia cinética, associada às moléculas de água presentes. Tal fato resulta na diminuição das forças de atração e, consequentemente, na saída de água do alimento, conduzindo a um decréscimo do conteúdo de umidade com a elevação da temperatura a uma dada atividade de água.

Tabela 2 - Parâmetros de ajuste das isotermas de sorção para os modelos matemáticos propostos para as duas formulações.

\begin{tabular}{|c|c|c|c|c|c|c|c|c|c|c|}
\hline & \multicolumn{5}{|c|}{ F1 } & \multicolumn{5}{|c|}{ F2 } \\
\hline Modelos & \multicolumn{3}{|c|}{ Parâmetros } & \multirow[t]{2}{*}{$\mathbf{R}^{2}$} & \multirow{2}{*}{$\begin{array}{c}\text { MRE } \\
(\%)\end{array}$} & \multicolumn{3}{|c|}{ Parâmetros } & \multirow[t]{2}{*}{$\mathbf{R}^{2}$} & \multirow{2}{*}{$\begin{array}{c}\text { MRE } \\
(\%)\end{array}$} \\
\hline GAB & $\mathbf{X m}$ & C & $\mathbf{K}$ & & & $\mathbf{X m}$ & C & $\mathbf{K}$ & & \\
\hline & 0,3438 & $1 \mathrm{E}+06$ & 0,8899 & 0,9181 & 4,255 & 0,4613 & 3,0984 & 0,4891 & 0,8486 & 12,073 \\
\hline \multirow[b]{2}{*}{ Oswin } & $\mathbf{a}$ & b & & & & $\mathbf{a}$ & b & & & \\
\hline & 0,6917 & 0,3255 & & 0,8635 & 1,879 & 0,2588 & 0,4012 & & 0,9353 & 1,835 \\
\hline \multirow[b]{2}{*}{ Halsey } & $\mathbf{A}^{\prime}$ & $\mathbf{r}$ & & & & $\mathbf{A}^{\prime}$ & $\mathbf{r}$ & & & \\
\hline & 0,2954 & 1,9704 & & 0,9127 & 2,485 & 0,0678 & 1,722 & & 0,9677 & 4,124 \\
\hline \multirow[b]{2}{*}{ BET } & b & c & & & & b & c & & & \\
\hline & 2,8782 & 0,028 & & 0,9571 & 5,473 & 6,837 & 0,3255 & & 0,9903 & 6,081 \\
\hline
\end{tabular}

\section{CONCLUSÃO}

Os biofilmes preparados pela mistura de pectina e MCB apresentaram propriedades melhoradas quando comparadas aos filmes preparados a partir de pectina pura, apresentando variabilidade na aparência e facilidade de remoção dos moldes. Para a produção futura de sacolas o ideal foi a utilização de altas concentrações de pectina, MCB e variação de glicerol. Esses filmes foram analisados em duas formulações com alto e baixo glicerol pela cinética de secagem com melhor ajuste para o modelo de Peleg e isotermas de sorção que teve melhor ajuste no modelo de Oswin. Bons ajustes foram encontrados além da variabilidade na aparência e facilidade de remoção dos moldes

\section{REFERENCIAS}

CARrAZA, L. R.; SILVA, M. L. D. A.; ÁVILA, J. C. C. Manual tecnológico de aproveitamento integral do fruto do babaçu. Brasília: Instituto Sociedade, População e Natureza (ISPN), 2012. SOUZA, C. R.

LIU, L., MARSHALL, L. F., HICKS, K. B. Pectin in controlled drug delivery - a review. Cellulose, v. 4, p. 15-24, 2007.

MADAMBA, P. S., DRISCOLL, R. H., BUCKLE, K. A. Thin layer drying characteristics of garlic slices. Journal of Food Engineering, v. 29, n. 1, p. 75-97, 1996.

SANTANA, A. A.; KIECKBUSCH, T. G. Physical evaluation of biodegradable films of calcium alginate plasticized with polyols, Brazilian Journal of Chemical Engineering, v. 30, p. $835-845,2013$. 